\section{Duregraph: A methodological approach to investigating duration in the photographic image}

\section{David van vliet $^{\mathrm{a}}$ \\ Marcos Mortensen Steagall ${ }^{\mathrm{b}}$}

Auckland University of Technology

This study elaborates on the methodological approach designed in a practice-led research that questioned how experienced time might be perceived in manipulated photographic images. The research was developed through a body of practice and exegetical writing that contributes to knowledge relating to time as duration and how it might be artistically exercised and embodied through photographic portraiture.

As creative practice grows inside academic inquiry, there is a need to determine an ongoing form of discourse and resources that support and expand on such methodologies. This study presents a contribution to that discourse and adds an overview of the practice with commentary about the development of the project. The website where the work is presented extends this discussion.

Because this research is produced within an artistic practice-led paradigm, it is essential to understand the methodology and methods adopted in its formation. Development of emerging outcomes is central to the research, and critical reflection permeates each point of the inquiry, driving decision making based on subjective experience.

The development of the research takes place in a cycle of four phases. In each phase, distinct and interrelated methods are used to develop and refine thinking. Phase one is concerned with ideation and the planning of activities; phase two involves the execution of the plan through shooting and experimenting in a photographic studio; in phase three the recorded data was processed. The fourth phase utilises targeted, strategic feedback that could impact on the refinements of the designs.

The inquiry is manifested through a series of five digital portraits that introduce subtle movements over time, while the subject remains within the frame. The resulting duregraphs constitute an unstable space between a photographic composite and a moving image, challenging conventions of power in viewing and expanding the way that time as duration might be conceived within digital photographic images.
Orcid number a 0000-0003-1480-3018

b $0000-0003-2108-4445$
KEYWORDS

Duration, Methodology, Photography, Practice-led research, Time 